\title{
Useful Background Information
}

\section{US presidents from Washington to Obama}

\begin{tabular}{|c|c|}
\hline & esident \\
\hline & George Washington (1732-99) \\
\hline & John Adams (1735-1826) \\
\hline 3 & Thomas Jefferson (1743-1826) \\
\hline & James Madison (1751-1836) \\
\hline & James Monroe (1758-1831) \\
\hline & John Quincy Adams (1767-1848) \\
\hline & Andrew Jackson (1767-1845) \\
\hline & Martin Van Buren (1782-1862) \\
\hline 9 & William Henry Harrison (1773-1841) \\
\hline 10 & John Tyler (1790-1862) \\
\hline & James K. Polk (1795-1849) \\
\hline & Zachary Taylor (1784-1850) \\
\hline & Millard Fillmore (1800-74) \\
\hline 14 & Franklin Pierce (1804-69) \\
\hline & James Buchanan (1791-1868) \\
\hline 16 & Abraham Lincoln (1809-65) \\
\hline & Andrew Johnson (1808-75) \\
\hline & Ulysses S. Grant (1822-85) \\
\hline 19 & Rutherford B. Hayes (1822-93) \\
\hline 20 & James A. Garfield (1831-81) \\
\hline & Chester A. Arthur (1830-86) \\
\hline & Grover Cleveland (1837-1908) \\
\hline & Benjamin Harrison (1833-1901) \\
\hline & Grover Cleveland (1837-1908) \\
\hline & William McKinley (1843-1901) \\
\hline 26 & Theodore Roosevelt (1858-1919) \\
\hline & William Howard Taft (1857-1930) \\
\hline & Woodrow Wilson (1856-1924) \\
\hline & Warren G. Harding (1865-1923) \\
\hline & Calvin Coolidge (1871-1933) \\
\hline & Herbert Hoover (1874-1964) \\
\hline & Franklin Delano Roosevelt (1882-194 \\
\hline & Harry S. Truman (1884-1972) \\
\hline
\end{tabular}

Party

Federalist

Federalist

Democratic-Republican

Democratic-Republican

Democratic-Republican

Democratic-Republican

Democrat

Democrat

Whig

Whig

Democrat

Whig

Whig

Democrat

Democrat

Republican

Union

Republican

Republican

Republican

Republican

Democrat

Republican

Democrat

Republican

Republican

Republican

Democrat

Republican

Republican

Republican

Democrat

Democrat
Term

1789-97

1797-1801

1801-09

1809-17

1817-25

1825-29

1829-37

1837-41

1841

1841-45

1845-49

1849-50

1850-53

1853-57

1857-61

1861-65

1865-69

1869-77

1877-81

1881

1881-85

1885-89

1889-93

1893-97

1897-1901

1901-09

1909-13

1913-21

1921-23

1923-29

1929-33

1933-45

1945-53 
34 Dwight D. Eisenhower (1890-1969)

35 John F. Kennedy (1917-63)

36 Lyndon B. Johnson (1908-73)

38 Gerald R. Ford (1913-2006)

39 Jimmy Carter (b. 1924)

40 Ronald Reagan (1911-2004)

41 George H. W. Bush (b. 1924)

42 William Jefferson Clinton (b. 1946)

43 George Walton Bush (b. 1946)

44 Barack Obama (b. 1961)
Republican

Democrat

Democrat

Republican

Democrat

Republican

Republican

Democrat

Republican

Democrat
$1953-61$

$1961-63$

1963-69

1974-77

1977-81

1981-89

1989-93

1993-2001

2001-09

2009

\section{Membership of the Supreme Court (2009)}

\begin{tabular}{|c|c|c|c|}
\hline Justice & Year of birth & Year of appointment & $\begin{array}{l}\text { President who made } \\
\text { the appointment }\end{array}$ \\
\hline \multicolumn{4}{|l|}{ Chief fustice } \\
\hline John Roberts & 1955 & 2005 & G. W. Bush \\
\hline \multicolumn{4}{|l|}{ Associate justices } \\
\hline John Stevens & 1920 & 1975 & G. Ford \\
\hline Antonin Scalia & 1936 & 1986 & R. Reagan \\
\hline Anthony Kennedy & 1936 & 1988 & R. Reagan \\
\hline Clarence Thomas & 1948 & 1991 & G. H. Bush \\
\hline Ruth Ginsburg & 1933 & 1993 & W. Clinton \\
\hline Stephen Breyer & 1938 & 1994 & W. Clinton \\
\hline Samuel Alito & 1950 & 2006 & G. W. Bush \\
\hline Sonia Sotomayor & 1954 & 2009 & B. Obama \\
\hline
\end{tabular}

N.B. As of September 2009, the average age of the nine justices is sixty-seven.

Party control of Congress (2009)

\begin{tabular}{lcc}
\hline Party & House & Senate \\
\hline Democrats & 256 & 57 \\
Independents & 0 & 2 \\
Republicans & 178 & 40 \\
Vacancies & 1 & 1 \\
\hline
\end{tabular}

Party control of state governorships (2009)

\begin{tabular}{ll}
\hline Party & Governorships held \\
\hline Democrats & 28 \\
Republicans & 22 \\
\hline
\end{tabular}

All figures correct as in September 2009. 